\title{
Radioimmunoscintigraphy for Postprostatectomy Radiotherapy: Analysis of Toxicity and Biochemical Control
}

\author{
Ashesh B. Jani, MD, MSEE${ }^{1}$; Michael J. Blend, $\mathrm{PhD}, \mathrm{DO}^{2}$; Russell Hamilton, $\mathrm{PhD}^{3}$; Charles Brendler, $\mathrm{MD}^{4}$; \\ Charles Pelizzari, $\mathrm{PhD}^{1}$; Lani Krauz, RN ${ }^{1}$; Bipin Sapra, MTech ${ }^{1}$; Srinivasan Vijayakumar, MD ${ }^{5}$; Azhar Awan, $\mathrm{MD}^{1}$; \\ and Ralph R. Weichselbaum, MD ${ }^{1}$ \\ ${ }^{I}$ Department of Radiation and Cellular Oncology, University of Chicago, Chicago, Illinois; ${ }^{2}$ Section of Nuclear Medicine, \\ Department of Radiology, University of Illinois, Chicago, Illinois; ${ }^{3}$ Department of Radiation Oncology, University of Arizona, \\ Tucson, Arizona; ${ }^{4}$ Section of Urology, Department of Surgery, University of Chicago, Chicago, Illinois; and ${ }^{5}$ Department of \\ Radiation Oncology, University of California, Davis, California
}

Our goal was to evaluate the role of radioimmunoscintigraphy (RIS) directed against prostate-specific membrane antigen (PSMA) in influencing postprostatectomy radiotherapy (RT) toxicity and biochemical control. Methods: The records of 107 postprostatectomy RT patients were reviewed. The group for whom no RIS scan was obtained (group A, $n=54$ ) was identified as was the group for whom a RIS scan was obtained (group B, $n=53$ ). Group B was further subdivided into those who had a RIS and CT-scan correlation to aid in treatment planning (subgroup B1, $n=40$ ) versus those who did not (subgroup B2, $n=13$ ). Gastrointestinal (Gl) and genitourinary (GU) toxicities were reviewed for each of these groups and subgroups and compared. Biochemical failures (defined as 2 successive PSA rises after a nadir of $\geq 0.2 \mathrm{ng} / \mathrm{mL}$ ) were identified to generate biochemical failure-free survival (BFFS) curves for each of the groups and subgroups. Results: No significant differences in late toxicity were observed between any group or subgroup. However, acute Gl toxicity was higher in group B versus group $\mathrm{A}(P=0.026)$, and acute $\mathrm{GU}$ toxicity was higher in subgroup B2 versus subgroup B1 $(P=0.050)$. Overall, most toxicity was grade 1 or 2 ; only one case of grade 3 toxicity and no cases of grade 4 or 5 toxicity were observed. Three-year BFFS was higher for group B versus group A ( $80.7 \%$ vs. $75.5 \%)$ and for subgroup B1 versus subgroup B2 (84.5\% vs. $71.6 \%)$. On multivariate analysis of pretreatment (age, race), surgical/ staging (stage, grade, margin status, extracapsular extension, lymph node status, seminal vesicle invasion, post-radical retropubic prostatectomy [RRP] prostate-specific antigen [PSA] nadir, maximum post-RRP PSA, and RRP-to-RT interval), and treatment (hormone therapy, RT dose, RT technique, RIS scan, and RIS/CT correlation) factors on BFFS, the only covariate reaching significance was RIS/CT correlation $(P=0.042)$. Conclusion: A small BFFS advantage was observed in patients for whom RIS was used to guide RT decision making and treatment planning; however, this advantage only reached significance in

\footnotetext{
Received Dec. 3, 2003; revision accepted Feb. 2, 2004.

For correspondence or reprints contact: Ashesh B. Jani, MD, MSEE, Department of Radiation and Cellular Oncology, University of Chicago Hospitals, 5758 S. Maryland Ave., MC 9006, Chicago, IL 60637.

E-mail: jani_1969@yahoo.com
}

this study for those for whom the RIS/CT correlation was used to guide target definition. The improved PSA control using RIS was achieved with a small increase in acute toxicity but with no observed change in late toxicity. These findings can serve as the basis for prospective studies in this area of investigation.

Key Words: prostate cancer; prostatectomy; radiotherapy; radioimmunoscintigraphy

J Nucl Med 2004; 45:1315-1322

$\mathbf{P}_{\mathrm{r}}$ rostate cancer is among the most common malignancies for which health care intervention is sought (1-3), and it is treated most commonly with radical retropubic prostatectomy (RRP) (4) or radical radiation therapy (RT) (5-6). For those patients who are assessed after RRP to have high-risk disease that is predictive of a local recurrence by pathologic findings or for those patients who likely have a local-only recurrence by prostate-specific antigen (PSA) record, clinical examination, or radiologic findings, post-RRP RT can be considered and has been used with success (7-16).

Radioimmunoscintigraphy (RIS), performed in the current study by targeting the prostate-specific membrane antigen (PSMA) (ProstaScint; Cytogen Corp.), has been studied in the diagnostic setting for prostate cancer (17-24). The sensitivity and specificity have been examined in a recent multiinstitutional trial (21) that documented the incidence of prostate fossa, pelvic nodes, and extrapelvic uptake among different clinical settings; the approximate values for diagnostic parameters in the postsurgery setting (the primary scenario in this study) are as follows: sensitivity, $75 \%$ (extraprostatic) and 92\% (prostate fossa); specificity, 86\%; positive predictive value, $81 \%$; and negative predictive value, $67 \%$.

RIS is useful in determining whether pelvic or extrapelvic lymphadenopathy exists before undertaking local or locoregional therapy. Recent efforts have evaluated the role of RIS 
in the setting of postprostatectomy RT decision making, and RIS was found to aid, by providing information complementary to that provided on the bone scan or abdominopelvic CT scan, in (a) guiding the decision to offer RT and (b) guiding the decision of the general treatment volume (prostate bed only vs. prostate bed and regional pelvic lymph nodes) (25). At our institution consortium in Chicago, we have integrated further RIS into the treatment planning process; we have developed and reported a technique to register the RIS scan with the RT treatment-planning CT scan using vessel registration (26-28), and we reported on how this technique was used to guide and to modify the definition of the prostate bed portion of the postprostatectomy clinical target volume (CTV) for external beam RT (29). Although there appears to be consensus on the diagnostic and technical aspects of RIS, the impact of RIS on clinical outcome is currently a matter of controversy. The survival advantage using RIS as a diagnostic study has been formally examined by several groups, with both positive (30) and negative studies (31) having been reported thus far.

The specific goals of the current study were (a) to determine the effects of RIS and the RIS/CT correlation on post-RRP RT acute and late gastrointestinal (GI) and genitourinary (GU) toxicity and (b) to determine the influence of RIS (particularly the RIS/CT image correlation specific to our institution) on the biochemical outcome of post-RRP RT patients.

\section{MATERIALS AND METHODS}

The patient population we studied consisted of 107 consecutive post-RRP prostate cancer patients who were treated in our hospital consortium between 1988 and 2002 and for whom treatment and follow-up information were available. The patient database was approved by the Institution Review Boards of all of the hospitals (University of Chicago, University of Illinois, and LaGrange Memorial Hospital) whose patient data were used for this investigation. Furthermore, because the current study is retrospective, a formal waiver of informed consent was requested and approved before conducting this study.

From the original cohort of 107 patients, a group consisting of 54 patients (group A) was identified, which comprised patients who had not had a RIS scan. The remaining 53 patients (group B) had a RIS scan; this group was further subdivided into the 40 patients who had the RIS/planning-CT correlation conducted (subgroup B1) versus those 13 patients who did not have such a correlation performed (subgroup B2). In subgroup B2, it should be noted that although the RIS/CT correlation was not performed, RIS was used as a diagnostic test to guide the RT decision and general treatment fields.

The patient and disease characteristics of these groups are shown in Table 1. This table displays the patient demographics (age, race), RRP pathologic findings (stage, grade, margin status, seminal vesicle invasion status, extracapsular extension, and lymph node status), and postprostatectomy course leading to RT consultation (post-RRP PSA nadir, post-RRP PSA follow-up course, hormone therapy, and interval from RRP to RT consultation). As demonstrated in Table 1, margin status and $\mathrm{T}$ stage were somewhat different among the groups; for this reason, these pa-
TABLE 1

Patient and Disease Characteristics Summary

Group A Group B Group B1 Group B2

\begin{tabular}{|c|c|c|c|c|}
\hline No. of patients & 54 & 53 & 40 & 13 \\
\hline \multicolumn{5}{|l|}{ Demographics } \\
\hline Age (y [mean]) & 63.3 & 57.8 & 50.0 & 60.3 \\
\hline \multicolumn{5}{|l|}{ Race } \\
\hline White & 24 & 33 & 26 & 7 \\
\hline African American & 29 & 16 & 11 & 5 \\
\hline Hispanic & 1 & 3 & 2 & 1 \\
\hline Other & 0 & 1 & 1 & 0 \\
\hline \multicolumn{5}{|l|}{ Prostatectomy findings } \\
\hline \multicolumn{5}{|l|}{ T stage } \\
\hline $\mathrm{pT} 1 / \mathrm{T} 2$ & 10 & 16 & 10 & 6 \\
\hline pT3 & 42 & 32 & 26 & 6 \\
\hline pT4 & 2 & 3 & 2 & 1 \\
\hline $\mathrm{pTx}$ & 0 & 2 & 2 & 0 \\
\hline \multicolumn{5}{|l|}{ Gleason score (GS) } \\
\hline GS 5 & 4 & 3 & 2 & 1 \\
\hline GS 6 & 15 & 12 & 7 & 5 \\
\hline GS 7 & 20 & 27 & 21 & 6 \\
\hline GS 8 & 4 & 5 & 5 & 0 \\
\hline GS 9 & 5 & 4 & 3 & 1 \\
\hline Not recorded & 6 & 2 & 3 & 0 \\
\hline \multicolumn{5}{|l|}{ Margins } \\
\hline Positive & 40 & 28 & 19 & 9 \\
\hline Negative & 12 & 23 & 19 & 4 \\
\hline Not recorded & 2 & 2 & 2 & 0 \\
\hline \multicolumn{5}{|l|}{ Seminal vesicle inv. } \\
\hline Positive & 15 & 15 & 13 & 2 \\
\hline Negative & 37 & 36 & 25 & 11 \\
\hline Not recorded & 2 & 2 & 2 & 0 \\
\hline \multicolumn{5}{|l|}{ Extracaps. extension } \\
\hline Yes & 33 & 32 & 26 & 6 \\
\hline No & 20 & 19 & 12 & 7 \\
\hline Not recorded & 1 & 2 & 2 & 0 \\
\hline \multicolumn{5}{|l|}{ Lymph nodes inv. } \\
\hline No & 42 & 47 & 37 & 10 \\
\hline Yes & 4 & 0 & 0 & 0 \\
\hline Not sampled & 8 & 6 & 3 & 3 \\
\hline \multicolumn{5}{|l|}{ Post-RRP PSA nadir } \\
\hline $\mathrm{PSA} \leq 0.1$ & 22 & 28 & 19 & 9 \\
\hline $0.1<\mathrm{PSA} \leq 0.2$ & 10 & 6 & 5 & 1 \\
\hline $0.2<\mathrm{PSA} \leq 0.3$ & 3 & 11 & 10 & 1 \\
\hline $0.3<\mathrm{PSA} \leq 0.5$ & 6 & 1 & 1 & 0 \\
\hline $0.5<\mathrm{PSA} \leq 1.0$ & 5 & 5 & 4 & 1 \\
\hline $1.0<\mathrm{PSA}$ & 7 & 2 & 1 & 1 \\
\hline Not recorded & 1 & 1 & 1 & 0 \\
\hline \multicolumn{5}{|l|}{ Highest PSA post-RRP } \\
\hline PSA $\leq 0.1$ & 13 & 7 & 3 & 4 \\
\hline $0.1<\mathrm{PSA} \leq 0.2$ & 8 & 7 & 5 & 2 \\
\hline $0.2<\mathrm{PSA} \leq 0.3$ & 4 & 5 & 5 & 0 \\
\hline $0.3<\mathrm{PSA} \leq 0.5$ & 6 & 6 & 5 & 1 \\
\hline $0.5<\mathrm{PSA} \leq 1.0$ & 9 & 12 & 9 & 3 \\
\hline $1.0<\mathrm{PSA} \leq 2.0$ & 7 & 11 & 10 & 1 \\
\hline $2.0<\mathrm{PSA}$ & 6 & 5 & 3 & 2 \\
\hline Not recorded & 1 & 0 & 0 & 0 \\
\hline \multicolumn{5}{|l|}{ RRP-to-RT interval (d) } \\
\hline Mean & 352 & 564 & 544 & 573 \\
\hline
\end{tabular}

$\mathrm{T}=$ tumor; inv. = invasion; Extracaps. = extracapsular. 
TABLE 2

Treatment Characteristics Summary

\begin{tabular}{lrrrr}
\hline & Group A & Group B & Group B1 & Group B2 \\
\hline $\begin{array}{l}\text { No. of patients } \\
\text { Volume }\end{array}$ & 54 & 53 & 40 & 13 \\
$\quad$ Whole pelvis initially & 9 & 6 & 4 & 2 \\
$\quad \begin{array}{l}\text { Prostate bed only } \\
\text { Technique }\end{array}$ & 45 & 47 & 36 & 11 \\
$\quad$ 4-Field & 41 & 21 & 16 & 5 \\
$\quad$ 6-Field & 8 & 21 & 13 & 8 \\
$\quad$ IMRT & 5 & 11 & 11 & 0 \\
Final dose & & & & \\
$\quad<60$ & 23 & 2 & 0 & 2 \\
$\quad 60-64$ & 8 & 5 & 5 & 0 \\
$\quad 64-66$ & 17 & 29 & 22 & 27 \\
$\quad$ M6 & 9 & 17 & 13 & 4 \\
$\quad$ Mean & $6,430.5$ & $6,644.4$ & $6,780.0$ & $6,677.5$ \\
Follow-up post-RT (d) & & & & \\
$\quad$ Mean & 814 & 648 & 661 & 673 \\
$\quad$ Median & 696 & 480 & 433 & 532 \\
$\quad$ Maximum & 3,561 & 2,080 & 2,268 & 1,536 \\
Hormone therapy & & & & \\
$\quad$ Yes & 6 & 29 & 22 & 6 \\
$\quad$ No & 46 & 24 & 18 & 7 \\
$\quad$ Not recorded & 1 & 0 & 0 & 0 \\
\hline
\end{tabular}

rameters were later analyzed using multivariate analysis (as were all pathologic features).

For each patient, a planning pelvic CT scan was obtained at the time of simulation using 5-mm spacing. Rectal and bladder contrasts were used to visualize these critical and avoidance structures. RIS scans, when conducted, were obtained in the nuclear medicine department, typically during the same week as the planning CT scan. The RIS scan had 2 components: a ${ }^{99 \mathrm{~m} T c-l a b e l e d ~ r e d ~ b l o o d}$ cell (RBC) SPECT scan and a simultaneously acquired ${ }^{111}$ Inlabeled monoclonal antibody (mAb) capromab pendetide (7E11.C5) RIS scan (26-29). For those patients having the RIS and CT registration performed, intravenous contrast was used during the planning CT scan to enable the vessel registration (26-29).

If the whole pelvis was treated, a 4-field technique was used for this portion; however, different techniques were used for the prostate bed RT: 4-field or 6-field conformal therapy or intensitymodulated radiotherapy (IMRT) (32). The treatment characteristics for these groups are shown in Table 2. As this table displays, a higher percentage of group A patients were treated with the older 4-field technique. In addition, a higher percentage of patients received hormone therapy in group B versus group A. Although this difference in percentages is a potential source of bias, hormone therapy and treatment technique were entered (as were all treatment characteristics) as covariates in the multivariate analysis, as described. In addition, hormone therapy, when administered, was short-term neoadjuvant or adjuvant therapy that affected the PSA time course only transiently and would not be expected to prohibit later biochemical failure analysis (33-34). The other treatment factors-radiation dose and general treatment volume-were similar between groups A and B and between subgroups B1 and B2. The radiation dose was selected according to a consensus conference examining this issue (35), which recommended approximately $64 \mathrm{~Gy}$.

For group B patients, the RIS reports were reviewed and tabulated. The RIS scans were read by one board-certified nuclear medicine physician who was involved in the initial development of the procedure as well as in several RIS clinical trials. Because only one physician read the RIS scans, interobserver variability in interpretation of the scans is not a confounding variable in this particular investigation. Although the authors recognize that having multiple observers reading would reduce potential errors of one reader, the experience of the reader and the later correlation with the clinical outcome make retroactive multiple readings inappropriate, as the toxicity and biochemical control analyses were analyzed as a function of administered therapy, which was guided by the single observer. Planar and volume SPECT datasets were obtained using a dual-head SPECT Prism 2000 Picker/Philips camera. This dual-energy procedure for acquisition of data and interpretation is described in considerable detail in a separate communication (22) and thus is not repeated here. The scans were read with knowledge of the patient's clinical history but not with the aid of CT or MRI information; in addition, CT and SPECT systems were not used. Of important note, the RIS/CT correlation described earlier was not a diagnostic test: The RIS scan was performed and read separately from the CT study, often several weeks before the planning CT scan. Thus, the RIS/CT correlation was not intended to be a novel diagnostic entity but rather a tool to assist the treatment-planning target design. This use of the RIS/CT correlation as a treatment-planning tool, which did involve several observers, is described in considerable detail in a prior article (29). For each patient, the RIS findings, with regard to uptake in the prostate fossa $(\mathrm{PF})$, pelvis $(\mathrm{P})$ (i.e., uptake within the pelvis in a region outside of the prostate fossa), or extrapelvic (EP) uptake, were reviewed. The summary of these findings, for group B1 versus group B2, is shown in Table 3 . The influence of the RIS findings on RT decision making at our institution has been previously reported (25). Except for one patient for whom EP uptake was viewed to be a false-positive, the presence of EP uptake usually caused the clinician to abort the decision to offer RT, and no treatment or follow-up information would be expected on these patients. In this manner, group B represents a selected patient population over that of group A, because the group B patients had the RIS scan done in addition to standard testing, including CT scan and bone scan when available. It is in this setting in which most previous RT studies analyzing RIS outcome analyses have been done $(30,31)$ (i.e., to determine the impact of RIS as a diagnostic test). One additional role that RIS had was in guiding the clinician in some cases to offer RT to the $\mathrm{PF}+\mathrm{P}$ group as opposed to the PF-only group (25).

TABLE 3

RIS Results Summary

\begin{tabular}{lccc}
\hline \multicolumn{1}{c}{ Variable } & Group B & Group B1 & Group B2 \\
\hline $\begin{array}{l}\text { No. of patients } \\
\text { Result }\end{array}$ & 53 & 40 & 13 \\
PF only & 40 & 32 & 9 \\
P only & 0 & 0 & 0 \\
EP only & 1 & 0 & 1 \\
PF + P & 10 & 7 & 3 \\
PF + EP & 1 & 1 & 0 \\
PF + P + EP & 0 & 0 & 0
\end{tabular}

$\mathrm{PF}=$ prostate fossa; $\mathrm{P}=$ pelvic node uptake; $\mathrm{EP}=$ extrapelvic uptake. 
TABLE 4

Acute Toxicity Analysis

\begin{tabular}{|c|c|c|c|c|c|c|c|c|}
\hline \multirow[b]{2}{*}{ Variable } & \multicolumn{4}{|c|}{ Rectum } & \multicolumn{4}{|c|}{ Bladder } \\
\hline & Group A & Group B & Subgroup B1 & Subgroup B2 & Group A & Group B & Subgroup B1 & Subgroup B2 \\
\hline Grade 0 & $21 / 54=39 \%$ & $15 / 52=29 \%$ & $12 / 39=31 \%$ & $3 / 13=23 \%$ & $18 / 54=33 \%$ & $16 / 52=31 \%$ & $13 / 39=33 \%$ & $3 / 13=23 \%$ \\
\hline Grade 1 & $11 / 54=20 \%$ & $19 / 52=37 \%$ & $13 / 39=33 \%$ & $4 / 13=31 \%$ & $25 / 54=46 \%$ & $25 / 52=48 \%$ & $21 / 39=54 \%$ & $4 / 13=31 \%$ \\
\hline Grade 2 & $21 / 54=39 \%$ & $18 / 52=35 \%$ & $14 / 39=36 \%$ & $6 / 13=46 \%$ & $11 / 54=20 \%$ & $11 / 52=21 \%$ & $5 / 39=13 \%$ & $6 / 13=46 \%$ \\
\hline Grade 3 & $1 / 54=2 \%$ & $0 / 52=0 \%$ & $0 / 39=0 \%$ & $0 / 13=0 \%$ & $0 / 54=0 \%$ & $0 / 52=0 \%$ & $0 / 39=0 \%$ & $0 / 13=0 \%$ \\
\hline Grade 4 & $0 / 54=0 \%$ & $0 / 52=0 \%$ & $0 / 39=0 \%$ & $0 / 13=0 \%$ & $0 / 54=0 \%$ & $0 / 52=0 \%$ & $0 / 39=0 \%$ & $0 / 13=0 \%$ \\
\hline Grade 5 & $0 / 54=0 \%$ & $0 / 52=0 \%$ & $0 / 39=0 \%$ & $0 / 13=0 \%$ & $0 / 54=0 \%$ & $0 / 52=0 \%$ & $0 / 39=0 \%$ & $0 / 13=0 \%$ \\
\hline$P$ & \multicolumn{2}{|c|}{$0.026(B>A)$} & \multicolumn{2}{|c|}{$0.720(\mathrm{~B} 1-\mathrm{B} 2)$} & \multicolumn{2}{|c|}{$0.923(\mathrm{~A}-\mathrm{B})$} & \multicolumn{2}{|c|}{$0.050(\mathrm{~B} 2>\mathrm{B} 1)$} \\
\hline
\end{tabular}

The acute and late toxicities were reviewed and tabulated for group A, group B, subgroup B1, and subgroup B2 according to the Radiation Therapy Oncology Group (RTOG) toxicity grading criteria (36-38) for all patients. Then, we made group and subgroup comparisons by using the $\chi^{2}$ test (39) to determine the influence of RIS and the influence of the RIS/CT correlation, respectively, on the toxicity outcomes.

In addition, we analyzed biochemical failure-free survival (BFFS). Using the definition of biochemical failure as 2 consecutive rises from PSA nadir to an absolute level greater than or equal to $0.2 \mathrm{ng} / \mathrm{mL}$ (with time of failure being declared midway between the nadir and the first PSA rise), we constructed Kaplan-Meier BFFS curves (40). Under this failure definition, those PSA levels that continually rise without a nadir are also counted as failures (effectively, the first follow-up after RT was taken to be the nadir in these cases). This definition combines features of definitions that rely on an absolute threshold and those that rely on successive rises (6-16). Survival curves were generated for the different groups and subgroups for qualitative comparison. Furthermore, a multivariate analysis, using the Cox proportional hazards model (40), was performed to quantitatively determine the influence of all pretreatment factors (age, race), surgical and staging factors (stage, grade, margin status, extracapsular extension, lymph node status, seminal vesicle invasion, post-RRP PSA nadir, maximum postRRP PSA, and RRP to RT interval), and treatment factors (hormone therapy, RT dose, RT technique, RIS scan, RIS findings, and RIS/CT correlation) on BFFS.

\section{RESULTS}

Tables 4 and 5 show the analyses for acute toxicity and late toxicity, respectively. As Table 4 demonstrates, there was a higher rate of acute GI toxicity in group A versus group B $\left(P=0.026, \chi^{2}\right)$; this was principally because of higher grade 1 toxicity (37\% vs. $20 \%$ ), shown in boldface in Table 4. In addition, there was a higher rate of GU toxicity in group B1 versus group B2 $\left(P=0.050, \chi^{2}\right)$; this was predominantly because of the higher grade 2 toxicity ( $46 \%$ vs. $13 \%$ ), again shown in boldface. As demonstrated, we observed no difference in acute GU toxicity between groups $\mathrm{A}$ and $\mathrm{B}$ and no difference in acute GI toxicity between subgroups B1 and B2. Table 5 demonstrates no difference in late GI or GU toxicity in any of the group or subgroup comparisons. As shown in the Tables 4 and 5, most of the observed toxicity was grade 1 or grade 2, the absolute incidence of grade 3 toxicity was low (only one case of acute GI toxicity was seen [in group A]), and we did not observe any grade 4 or grade 5 toxicity.

Figure 1 shows the BFFS curves for the various group and subgroup comparisons: Figure 1A displays the BFFS curves for group B versus group A; this comparison demonstrates the overall influence of RIS on biochemical control. As shown, 3-y BFFS for group B versus group A was $80.7 \%$ versus $75.5 \%$. Figure 1B displays the BFFS curves for subgroup B1 versus subgroup B2; this comparison demonstrates the influence, within the group that underwent the RIS scan, of the RIS/CT correlation on biochemical control. As shown, 3-y BFFS for subgroup B1 versus subgroup B2 was $84.5 \%$ versus $71.6 \%$. Figure $1 \mathrm{C}$ displays the BFFS curves for subgroup B1 versus group A; this comparison

TABLE 5

Late Toxicity Analysis

\begin{tabular}{|c|c|c|c|c|c|c|c|c|}
\hline \multirow[b]{2}{*}{ Variable } & \multicolumn{4}{|c|}{ Rectum } & \multicolumn{4}{|c|}{ Bladder } \\
\hline & Group A & Group B & Group B1 & Group B2 & Group A & Group B & Group B1 & Group B2 \\
\hline Grade 0 & $29 / 52=56 \%$ & $30 / 46=65 \%$ & $22 / 33=67 \%$ & $8 / 13=62 \%$ & $24 / 52=46 \%$ & $26 / 46=57 \%$ & $18 / 33=55 \%$ & $7 / 13=54 \%$ \\
\hline Grade 1 & $18 / 52=35 \%$ & $13 / 46=28 \%$ & $7 / 33=21 \%$ & $5 / 13=38 \%$ & $17 / 52=33 \%$ & $12 / 46=26 \%$ & $10 / 33=30 \%$ & $3 / 13=23 \%$ \\
\hline Grade 2 & $5 / 52=10 \%$ & $3 / 46=7 \%$ & $4 / 33=12 \%$ & $0 / 13=0 \%$ & $9 / 52=17 \%$ & $8 / 46=17 \%$ & $5 / 33=15 \%$ & $3 / 13=23 \%$ \\
\hline Grade 3 & $0 / 52=0 \%$ & $0 / 46=0 \%$ & $0 / 33=0 \%$ & $0 / 13=0 \%$ & $2 / 52=4 \%$ & $0 / 46=0 \%$ & $0 / 33=0 \%$ & $0 / 13=0 \%$ \\
\hline Grade 4 & $0 / 52=0 \%$ & $0 / 46=0 \%$ & $0 / 33=0 \%$ & $0 / 13=0 \%$ & $0 / 52=0 \%$ & $0 / 46=0 \%$ & $0 / 33=0 \%$ & $0 / 13=0 \%$ \\
\hline Grade 5 & $0 / 52=0 \%$ & $0 / 46=0 \%$ & $0 / 33=0 \%$ & $0 / 13=0 \%$ & $0 / 52=0 \%$ & $0 / 46=0 \%$ & $0 / 33=0 \%$ & $0 / 13=0 \%$ \\
\hline$P$ & \multicolumn{2}{|c|}{$0.375(\mathrm{~A}=\mathrm{B})$} & \multicolumn{2}{|c|}{$0.178(\mathrm{~B} 1=\mathrm{B} 2)$} & \multicolumn{2}{|c|}{$0.327(A=B)$} & \multicolumn{2}{|c|}{$0.722(\mathrm{~B} 1=\mathrm{B} 2)$} \\
\hline
\end{tabular}




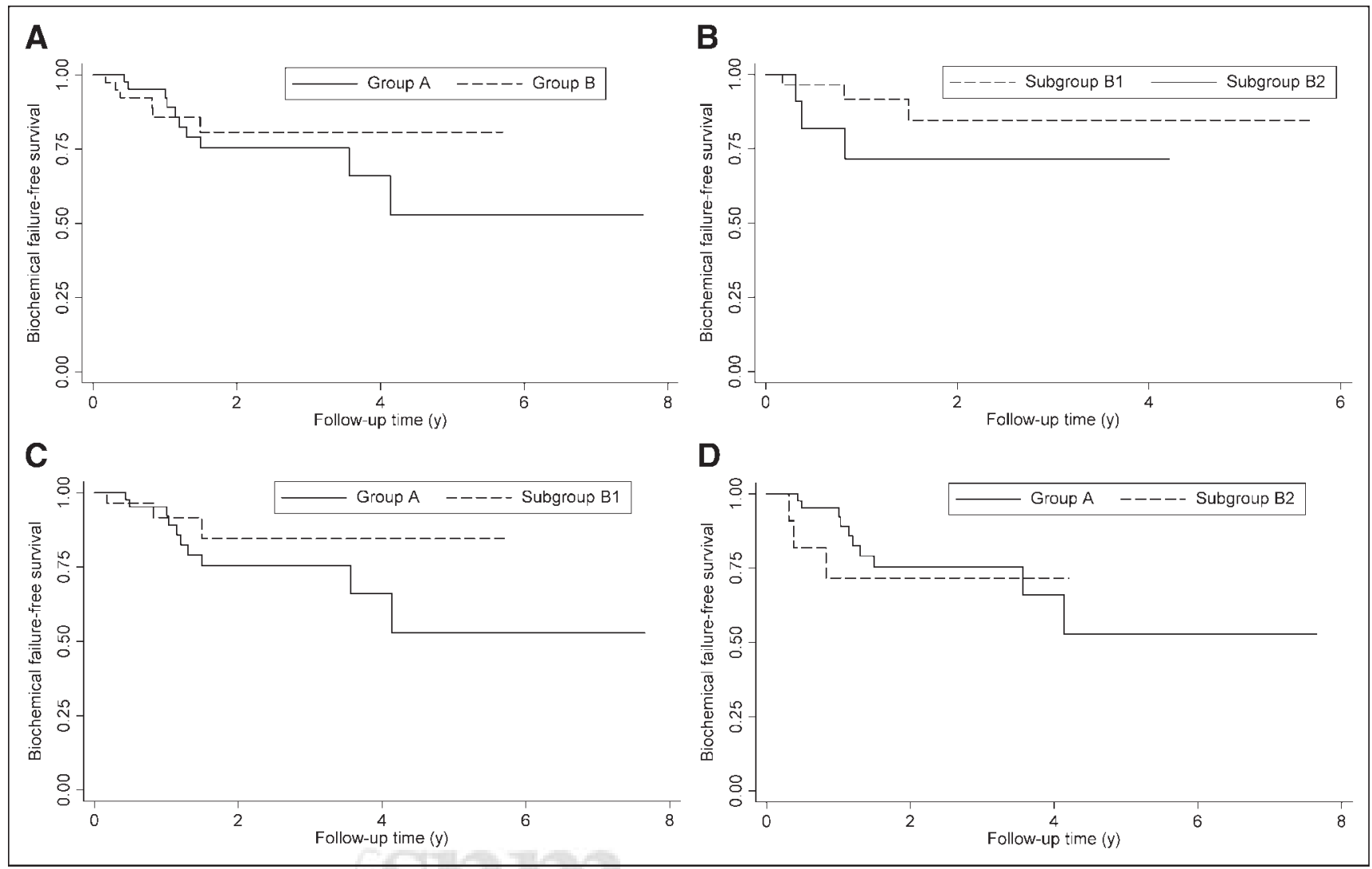

FIGURE 1. Kaplan-Meier BFFS curves: group A vs. group B (A); subgroup B1 vs. subgroup B2 (B); group A vs. subgroup B1 (C); group A vs. subgroup B2 (D).

displays biochemical control in the group undergoing the RIS/CT correlation against the comparison group not undergoing RIS. As shown, 3-y BFFS for subgroup B1 versus group A was $84.5 \%$ versus $75.5 \%$. Last, Figure 1D displays the BFFS curves for subgroup B2 versus group A; this comparison demonstrates the biochemical control in the subgroup undergoing RIS (but not undergoing the RIS/CT correlation) against the comparison group not undergoing RIS. As shown, 3-y BFFS for subgroup A versus subgroup B2 was $75.5 \%$ versus $71.6 \%$. It should be emphasized that although the overall cohort was well-powered (with $>100$ patients), this statistical power was diminished when the groups were broken into groups and subgroups. For this reason, meaningful pairwise statistical comparisons among the different groups and subgroups would not be adequately interpretable, and the pairwise results described above are intended to be for qualitative purposes only.

The qualitative results of the group and subgroup comparisons in Figure 1 were extended quantitatively by performing a multivariate analysis of pretreatment factors, surgical and staging factors, and treatment factors on biochemical outcome. The results of this multivariate analysis are shown in Table 6. It is noteworthy that although RIS scan is a covariate (representing the group A versus group B comparison), as is the RIS/CT correlation (representing the subgroup B1 vs. group A + subgroup B2 comparison), RIS findings cannot be an independent covariate because it is colinear with RIS scan when performing a multivariate analysis. Additionally, the results for lymph node status, which was included as a covariate in the multivariate analysis, are not displayed because of the degeneracy in the

TABLE 6

Multivariate Analyses of Factors Influencing BFFS

\begin{tabular}{llc}
\hline & \multicolumn{2}{c}{ Multivariate analysis } \\
\cline { 2 - 3 } \multicolumn{1}{c}{ Factor } & \multicolumn{1}{c}{ Hazard ratio } \\
Ag5\% confidence interval] & $P$ \\
\hline Race & $1.031[0.881,1.207]$ & 0.701 \\
T stage & $0.449[0.074,2.715]$ & 0.383 \\
Grade (Gleason score) & $0.178[0.005,6.126]$ & 0.339 \\
Margins & $2.771[0.794,9.663]$ & 0.110 \\
Seminal vesicle inv. & $0.181[0.003,10.732]$ & 0.412 \\
ECE & $0.405[0.406,4.034]$ & 0.441 \\
Post-RRP PSA nadir & $0.930[0.057,14.951]$ & 0.959 \\
Highest post-RRP PSA & $1.289[0.485,3.421]$ & 0.611 \\
RRP-to-RT interval & $0.911[0.359,2.310]$ & 0.845 \\
Treatment technique & $1.004[0.999,1.008]$ & 0.096 \\
Final RT dose & $1.762[0.250,12.393]$ & 0.569 \\
Hormone therapy & $0.998[0.994,1.001]$ & 0.194 \\
RIS scan & $8.836[0.023,3,268.082]$ & 0.470 \\
RIS/CT correlation & $1.209[0.030,48.349]$ & 0.920 \\
& $0.010[0.0001,0.848]$ & 0.042 \\
& & \\
\hline inv. = invasion. & & \\
\hline
\end{tabular}


absence of event rates in the small number of patients having node-positive disease. As displayed in Table 6, the only covariate reaching significance among all the pretreatment factors (age, race), surgical and staging factors (stage, grade, margin status, extracapsular extension, seminal vesicle invasion, post-RRP PSA nadir, maximum post-RRP PSA, and RRP to RT interval), and treatment factors (hormone therapy, RT dose, RT technique, RIS scan, and RIS/CT correlation) displayed was the RIS/CT correlation $(P=0.042)$.

\section{DISCUSSION}

Previous reports of the role of RIS in RT document the role of RIS as a diagnostic test (17-24,30-31). At our institution, we have tried to better understand the role of RIS as it relates to post-RRP RT by (a) describing the influence of RIS on RT decision making (25) and (b) analyzing the role of a RIS/CT correlation in designing the RT target (29). The current work extends these investigations by providing toxicity and biochemical control data of the population who underwent RIS and a comparison group who did not undergo RIS. The current efforts also add to the growing body of data examining the outcome after using RIS to guide prostate RT in different settings, such as brachytherapy (41).

Several observations about the toxicity analysis deserve mention. First, it appears as though the group who underwent RIS displayed higher grade 1 GI toxicity than those who did not undergo RIS. Also, the subgroup who underwent the RIS/CT correlation displayed higher grade $2 \mathrm{GU}$ toxicity than the subgroup who did not undergo the correlation. The reason for the higher toxicity in these cases may be related to the larger volume of prostate bed receiving a higher dose. A previous investigation found that the larger volumes would be expected to impact on the bladder dosevolume histograms (DVHs), but impact on the rectal DVHs in this investigation was higher than that predicted (29). In any case, the incidence of toxicity was low in absolute terms (as described, only one case of grade 3 toxicity was observed and no grade 4 or 5 toxicity was observed) and should not prohibit prospective investigation of RIS or RIS/CT correlation. Another fundamental issue is whether the increase in acute toxicity is acceptable in the face of the small BFFS advantage. That the late toxicity was not found to be significantly different between any group or subgroup is suggestive that the long-term outcome benefit/harm ratio is acceptable. However, this question, both for the acute as well as for the late toxicity, can only be quantitatively explored in the prospective setting, with tools such as quality-adjusted time without symptoms and toxicity (QTWiST) (42).

Several qualitative observations about the biochemical control analysis also bear mention. As Figure 1A describes, there was a small BFFS advantage to the overall use of RIS. On closer examination, Figure 1B shows that within the group receiving RIS, the subgroup undergoing the RIS/CT correlation had higher biochemical control than those who did not. Figure 1C, in addition, shows the higher survival curve of the subgroup undergoing RIS/CT correlation compared with the comparison group who did not undergo RIS. The biologic implications of this finding are that performing the RIS/CT correlation potentially allowed for better encompassing of the regions at risk. It should be emphasized, though, that as described in the Results section, the pairwise survival curve comparisons shown in Figure 1 were intended to be only qualitative comparisons, and as such, a statistically significant BFFS advantage cannot be shown in the subgroups.

Also bearing mention is the results of Figure 1D, which display similar curves between those RIS patients not undergoing RIS/CT correlation and the comparison group not undergoing RIS. These curves look very similar, implying that RIS did not assist in improving the survival rate in and of itself (i.e., without the correlation). This may be because, in this study, the RIS findings were acted on (i.e., uptake to $\mathrm{PF}+\mathrm{P}$ often involved increasing the RT fields to encompass this disease). This agrees with some previous reports documenting the absence of difference of RIS in assisting biochemical control when used as a diagnostic test (30) but does not agree with other studies that demonstrated such a difference (31). Caution is warranted in interpreting the conclusions in this subgroup versus the comparison group, because the study was underpowered to answer this question and, more important, not designed to answer this question formally. Indeed, for this reason, as well as the colinearity with RIS scan as described in the Methods section, RIS findings were not analyzed as an independent covariate.

In our investigation, the vast majority of patients undergoing RIS underwent the RIS/CT correlation specific to our institution. Over the past several years, several radiation treatment-planning software packages have been introduced that can perform multimodality registration; although in this study we used our institution-developed software, similar clinical results could be expected with any of several commercially available registration techniques. Of particular note, all pretreatment and treatment factors were not significant on multivariate analysis other than RIS/CT correlation. Even factors such as Gleason score and RT dose (which were fairly balanced between the different arms) and hormone therapy (which was given more commonly in group B than group A) did not reach significance, suggesting that these factors did not (within limitations of statistical power of this particular study) influence survival.

Because using RIS in the post-RRP RT setting is a relatively recent development, the follow-up for group B is predictably shorter than for group A. Although the current analysis does compare group B with the entire available length of follow-up in group A, because prostate cancer has a long natural history, longer follow-up is needed to see if the results described herein are maintained. However, it should be noted that the median follow-up of the study, 
particularly that of group A, is similar to that of many investigations examining post-RRP RT.

The definition of biochemical failure used in this investigation was, as described, a hybrid between those definitions relying on an absolute PSA threshold and those relying on successive PSA rises. Both of these general approaches have been used, and no consensus has been reached on the standard definition of failure in the post-RRP population. Within the successive rises approach, investigators have reported results using 3 successive rises or 2 successive rises. Within the absolute threshold approach, the threshold has varied among various reports from 0.2 to $1.0(6-16)$. Although absolute failure rate is, of course, dependent on the definition of failure, the results of the current study are less sensitive to this issue, as the same definition was applied to all groups, so the relative difference in failures would not be expected to change significantly as a function of the definition of failure.

We understand the biases and limitations inherent to the retrospective nature of the analyses reported here, but within these limitations the current investigation sheds light on the role of RIS in the post-RRP setting. The conclusion for the clinician is that the RIS appears to be of use to assist the post-RRP RT process but, if used, should be correlated with the planning-CT scan to maximize its benefit. We hope that the current study can provide a preliminary framework on which to design a prospective investigations in this area.

\section{CONCLUSION}

A small BFFS advantage was observed in patients in whom RIS was used to guide RT decision making and treatment planning; however, this advantage only reached significance in this study for those for whom RIS/CT correlation was used to guide target definition. The improved PSA control using RIS was achieved with a small increase in acute toxicity but with no observed change in late toxicity. These findings can serve as the basis for prospective studies in this area of investigation.

\section{ACKNOWLEDGMENT}

This work was funded in part by Cytogen Corp. (Princeton, NJ). None of the authors has financial interest in this work or any other conflicts of interest.

\section{REFERENCES}

1. Gronberg H. Prostate cancer epidemiology. Lancet. 2003;361:859-864.

2. Potosky AL, Feuer EJ, Levin DL. Impact of screening on incidence and mortality of prostate cancer in the United States. Epidemiol Rev. 2001;23:181-186.

3. Neal DE, Donovan JL. Prostate cancer: to screen or not to screen? Lancet Oncol. 2000;1:17-24

4. Sokoloff $\mathrm{MH}$, Brendler $\mathrm{CB}$. Indications and contraindications for nerve-sparing radical prostatectomy. Urol Clin North Am. 2001;28:535-543.

5. Jani AB, Hellman S. Early prostate cancer: clinical decision-making. Lancet. 2003;361:1045-1053.

6. Hanlon AL, Hanks GE. Failure patterns and hazard rates for failure suggest the cure of prostate cancer by external beam radiation. Urology. 2000;55: 725-729.

7. Anscher MS, Clough R, Dodge R. Radiotherapy for a rising prostate-specific antigen after radical prostatectomy: the first 10 years. Int J Radiat Oncol Biol Phys. 2000;48:369-375.

8. Schild SE, Buskirk SJ, Wong WW, et al. The use of radiotherapy for patients with isolated elevation of serum prostate specific antigen following radical prostatectomy. J Urol. 1996;156:1725-1729.

9. Petrovich Z, Lieskovsky G, Langholz B, et al. Postoperative radiotherapy in 423 patients with pT3N0 prostate cancer. Int J Radiat Oncol Biol Phys. 2002;53: 600-609.

10. Kalapurakal JA, Huang CF, Neriamparampil MM, et al. Biochemical disease-free survival following adjuvant and salvage irradiation after radical prostatectomy. Int J Radiat Oncol Biol Phys. 2002;54:1047-1054.

11. Duchesne GM, Dowling C, Frydenberg M, et al. Outcome, morbidity, and prognostic factors in post-prostatectomy radiotherapy: an Australian multicenter study. Urology. 2003;61:179-183.

12. Do T, Parker RG, Do C, et al. Salvage radiotherapy for biochemical and clinical failures following radical prostatectomy. Cancer J Sci Am. 1998;4:324-330.

13. Jacobson GM, Smith JA Jr, Stewart JR. Postoperative radiation therapy for pathologic stage C prostate cancer. Int J Radiat Oncol Biol Phys. 1987;13:10211024.

14. Carroll P. Rising PSA after a radical treatment. Eur Urol. 2001;40(suppl 2):9-16.

15. Wieder JA, Soloway MS. Incidence, etiology, location, prevention and treatment of positive surgical margins after radical prostatectomy for prostate cancer. J Urol. 1998;160:299-315.

16. Macdonald OK, Schild SE, Vora SA, et al. Radiotherapy for men with isolated increase in serum prostate specific antigen after radical prostatectomy. $J$ Urol. 2003; 170:1833-1837.

17. Manyak MJ, Hinkle GH, Olsen JO, et al. Immunoscintigraphy with indium-111capromab pendetide: evaluation before definitive therapy in patients with prostate cancer. Urology. 1999;54:1058-1063.

18. Quintana JC, Blend MJ. The dual-isotope ProstaScint imaging procedure: clinical experience and staging results in 145 patients. Clin Nucl Med. 2000;25:33-40.

19. Hinkle GH, Burgers JK, Neal CE, et al. Multicenter radioimmunoscintigraphic evaluation of patients with prostate carcinoma using indium-111 capromab pendetide. Cancer. 1998;83:739-747.

20. Murphy GP, Elgamal AA, Troychak MJ, et al. Follow-up ProstaScint scans verify detection of occult soft-tissue recurrence after failure of primary prostate cancer therapy. Prostate. 2000;42:315-317.

21. Sodee DB, Malguria N, Faulhaber P, et al. Multicenter ProstaScint imaging findings in 2154 patients with prostate cancer: The ProstaScint Imaging Centers. Urology. 2000;56:988-993.

22. Blend MJ, Sodee DB. ProstaScint: an update. In: Freeman LM, ed. Nuclear Medicine Annual. Philadelphia, PA: Lippincott Williams \& Wilkins; 2001:109142.

23. Feneley MR, Jan H, Granowska M, et al. Imaging with prostate-specific membrane antigen (PSMA) in prostate cancer. Prostate Cancer Prostatic Dis. 2000; $3: 47-52$.

24. Raj GV, Partin AW, Polascik TJ. Clinical utility of indium 111-capromab pendetide immunoscintigraphy in the detection of early, recurrent prostate carcinoma after radical prostatectomy. Cancer. 2002;94:987-996.

25. Jani AB, Blend MJ, Hamilton R, et al. Influence of radioimmunoscintigraphy on postprostatectomy radiotherapy treatment decision making. J Nucl Med. 2004; 45:571-578.

26. Hamilton RJ, Blend MJ, Pelizzari CA, Milliken BD, Vijayakumar S. Using vascular structure for CT-SPECT registration in the pelvis. J Nucl Med. 1999; 40:347-351.

27. Hamilton RJ, Blend MJ, Blend D, et al. Use of ProstaScint CT image registration in the post-operative radiation therapy of prostate cancer. Int J Radiat Oncol Biol Phys. 2000;38(suppl):358-359.

28. Sychra JJ, Lin KQ, Blend MJ. Detection of metastatic prostate cancer with simultaneous dual radioisotope SPECT images. RSNA [serial online]. 1997;1. Available at: http://ej.rsna.org/EJ_0_96/0034-97.fin/prostate.htm

29. Jani AB, Spelbring D, Hamilton R, et al. Impact of radioimmunoscintigraphy on definition of clinical target volume for radiotherapy after prostatectomy. $\mathrm{J} \mathrm{Nucl}$ Med. 2004;45:238-246.

30. Kahn D, Williams RD, Haseman MK, et al. Radioimmunoscintigraphy with In-111-labeled capromab pendetide predicts prostate cancer response to salvage radiotherapy after failed radical prostatectomy. J Clin Oncol. 1998;16: 284-289. 
31. Thomas CT, Bradshaw PT, Pollock BH, et al. Indium-111-capromab pendetide radioimmunoscintigraphy and prognosis for durable biochemical response to salvage radiation therapy in men after failed prostatectomy. J Clin Oncol. 2003;21:1715-1721.

32. Jani AB, Roeske JC, Rash C. Intensity modulated radiotherapy (IMRT) for prostate cancer. Clin Prostate Cancer. 2003;2:98-105.

33. Pilepich MV, Winter K, John MJ, et al. Phase III radiation therapy oncology group (RTOG) trial 86-10 of androgen deprivation adjuvant to definitive radiotherapy in locally advanced carcinoma of the prostate. Int J Radiat Oncol Biol Phys. 2001;50:1243-1252.

34. Eulau SM, Tate DJ, Stamey TA, Bagshaw MA, Hancock SL. Effect of combined transient androgen deprivation and irradiation following radical prostatectomy for prostatic cancer. Int J Radiat Oncol Biol Phys. 1998;41:735-740.

35. Cox JD, Gallagher MJ, Hammond EH, Kaplan RS, Schellhammer PF. Consensus statements on radiation therapy of prostate cancer: guidelines for prostate re-biopsy after radiation and for radiation therapy with rising prostate-specific antigen levels after radical prostatectomy. J Clin Oncol. 1999; 17:1155-1163.
36. Michalski JM, Purdy JA, Winter K, et al. Preliminary report of toxicity following 3D radiation therapy for prostate cancer on 3DOG/RTOG 9406. Int J Radiat Oncol Biol Phys. 2000;46:391-402.

37. Lawton CA, Won M, Pilepich MV, et al. Long-term treatment sequelae following external beam irradiation for adenocarcinoma of the prostate: analysis of RTOG studies 7506 and 7706. Int J Radiat Oncol Biol Phys. 1991;21:935-939.

38. Ryu JK, Winter K, Michalski JM, et al. Interim report of toxicity from 3D conformal radiation therapy (3D-CRT) for prostate cancer on 3DOG/RTOG 9406, level III (79.2 Gy). Int J Radiat Oncol Biol Phys. 2002;54:1036-1046.

39. Tello R, Crewson PE. Hypothesis testing II: means. Radiology. 2003;227:1-4.

40. Fleming TR, Lin DY. Survival analysis in clinical trials: past developments and future directions. Biometrics. 2000;56:971-983.

41. Ellis RJ, Vertocnik A, Kim E, et al. Four-year biochemical outcome after radioimmunoguided transperineal brachytherapy for patients with prostate adenocarcinoma. Int J Radiat Oncol Biol Phys. 2003;57:362-370.

42. Feldstein ML. Quality-of-life-adjusted survival for comparing cancer treatments. A commentary on TWiST and Q-TWiST. Cancer. 1991;67(suppl 3):851-854. 